\title{
TRANSANULAR INTERACTION IN [2.2]PHANES: MODELS FOR DIMERS?
}

\author{
W. GOLDACKER, K.H. HAUSSER, D. SCHWEITZER \\ and H.A. STAAB \\ Max-Planck-Institut, Abteilung für Molekulare Physik, Abteilung für Organische Chemie, \\ Jahn-Straße 29, 6900 Heidelberg, Fed. Rep. Germany
}

\begin{abstract}
The emission spectra of pseudo-ortho 3 and pseudo-geminal-4,7,12,15-tetramethoxy[2.2]paracyclophane $\underline{3}$ were measured in glass matrices at $1.3 \mathrm{~K}$. Furthermore, the zero field splitting parameters $D$ and $E$ and the decay rate constants $k_{i}$ of the excited triplet state were studied by optical detection of magnetic resonance in zero field. The results were compared with the corresponding monomer 1,4-dimethyl-2,5-dimethoxybenzene 1 .
\end{abstract}

\section{Introduction}

In several preceding papers [1-6] we reported our studies of the transanular $\pi$-electron interaction in a number of [2.2]phanes. The [2.2]phanes are molecules in which two aromatic units are kept in a face-to-face arrangement with a fixed distance by methylene bridges. In this paper we report the emission spectra and the zero field splitting parameters $D$ and $E$ and the decay constants $k_{t}$ of the two stereo-isomeric 4,7,12,15-tetramethoxy[2.2]paracyclophanes, the pseudo-ortho compound 2 and the pseudo-geminal compound 3, (see fig. 1) [7], and for the comparison the corresponding values of the monomer 1,4-dimethyl-2,5dimethoxybenzene. The investigation of the two stereoisomers with different but fixed orientations of the two subunits of the phane with respect to each other provides information about the influence of the orientation on the physical properties. The optical spectra and the optical detection of magnetic resonance (ODMR) were measured on glass matrices at $1.3 \mathrm{~K}$.

\section{Results}

Fig. 1 shows the fluorescence $(\mathrm{F})$ - and phosphorescence $(\mathrm{P})$-spectra of 1,2 and 3 in low concentration in glasses. In contrast to the sharply structured fluorescence and phosphorescence of most monomers investigated previously $[1,4,5]$, we find for $\underline{1}$ a broad-band structureless fluorescence and a well-structured phosphorescence with a broad underground. 


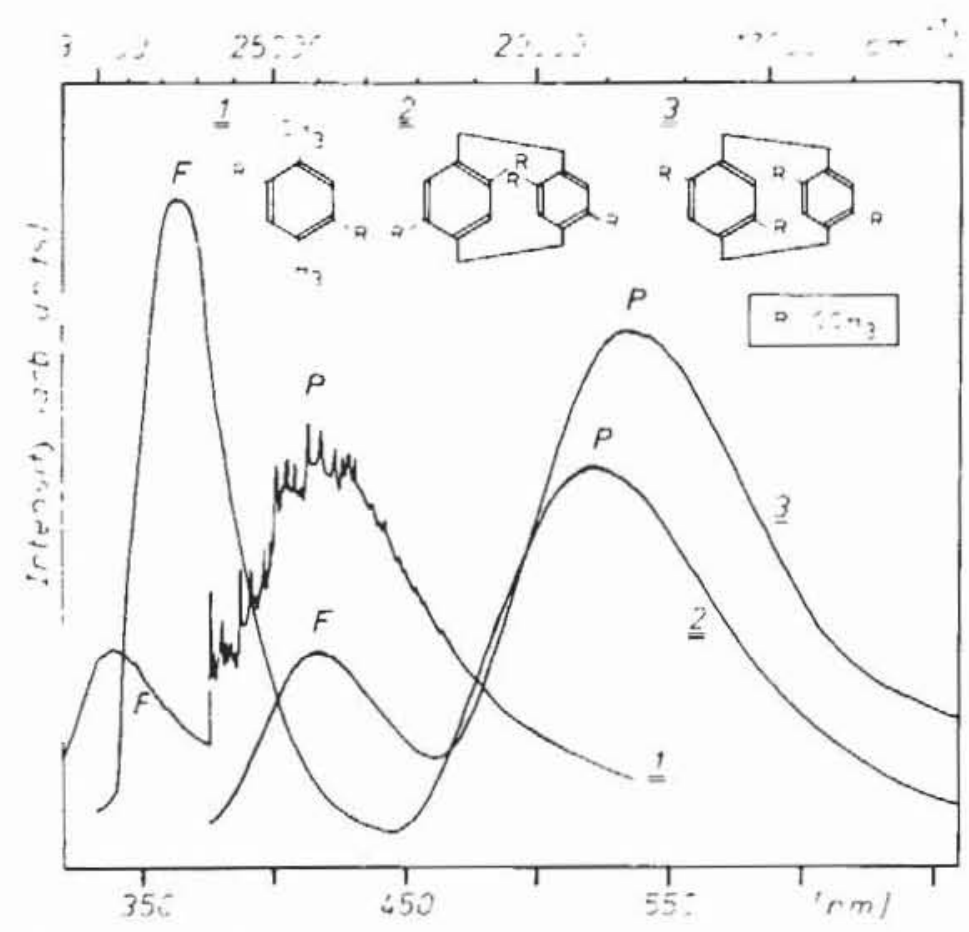

Fig. 1. Fluorescence $(\mathrm{F})$ and phosphorescence $(\mathrm{P})$ spectra of 1,4-dimethyl-2,5-dimethoxybenzene ( $I$ ) in n-octane, pseudo-ortho 4,7,12,15-tetramethoxy[2.2] paracyclophane (2) and pseudo-geminal[4,7,12,15tetramethoxy[2.2] paracyclophane $\underline{3}$ in 2 methyltetrahydrofurane (MTHF) at $1.3 \mathrm{~K}$.

Table 1

Zero field splitting parameters $|D|$ and $|E|$, total triplet sublevel decay rates $k_{1}$ and decay rate $\rho=\frac{1}{2} \Sigma_{1} k_{1}$ of $\underline{1}$ in n-octane, $\underline{2}$ and $\underline{3}$ in 2 methyltetrahydrofurane (MTHF)

\begin{tabular}{ccll}
\hline Molecule & \multicolumn{1}{c}{$\underline{\underline{2}}$} & \multicolumn{1}{c}{$\underline{\underline{3}}$} & \multicolumn{1}{c}{$\underline{-}$} \\
\hline$|D|\left[\mathrm{cm}^{-1}\right]$ & 0.1143 & 0.0983 & 0.0901 \\
$|E|\left[\mathrm{cm}^{-1}\right]$ & 0.0351 & 0.0287 & 0.0226 \\
$k_{x}\left[\mathrm{~s}^{-1}\right]$ & $0.11 \pm 0.01$ & $0.83 \pm 0.2$ & $5.5 \pm 0.5$ \\
$k_{y}\left[\mathrm{~s}^{-1}\right]$ & $0.67 \pm 0.05$ & $5.3 \pm 0.8$ & $6.7 \pm 0.9$ \\
$k_{z}\left[\mathrm{~s}^{-1}\right]$ & $0.16 \pm 0.03$ & $3.3 \pm 0.4$ & $1.8 \pm 0.2$ \\
$\rho\left[\mathrm{s}^{-1}\right]$ & 0.31 & 3.14 & 4.67 \\
\hline
\end{tabular}

The spectra of $\underline{1}$, dissolved in a n-octane or a 2-methyl-tetrahydrofurane glass, were found to be independent of the concentration within the range of $10^{-5}<c<$ $10^{-2} \mathrm{~mol} / \mathrm{l}$.

Table 1 shows the values of the zero-field splitting parameters $D$ and $E$ of the monomer $\underline{1}$ and of the two stereoisomer phanes $\underline{2}$ and $\underline{3}$. Furthermore, it gives the decay rates $k_{1}$ of the three triplet sublevels of these molecules as well as the average decay rate $\rho=\frac{1}{3} \Sigma_{1} k_{1}$.

\section{Discussion}

We interpret the width and lack of structure of the fluorescence band of $\underline{l}$ as resulting from strong electron-phonon coupling, i.e. the glasses used are not 
good Shpolskii matrices for $\underline{1}$. An alternative explanation of an intermolecular interaction within microcrystalline aggregates can probably be ruled out because of the absence of a concentration dependence even for concentrations as low as $10^{-5} \mathrm{~mol} / \mathrm{l}$.

In a previous paper [6] we have discussed the interaction between two identical subunits of a [2.2]phane. The stronger the interaction between the two subunits the larger becomes the parameter $\lambda$ which measures the contribution of the charge transfer terms to the total wave function. The crucial quantity for understanding the $\pi \pi$-interaction in phanes is the total intersystem bond density $d_{i}^{\pi}=\Sigma_{i j} d_{i j}^{\pi}$ in the excited singlet and triplet state, that is the sum over the different bond densities of a $\pi$-orbital at one atom $i$ in one subunit and a $\pi$-orbital at an atom $j$ in the other subunit (eq. (2) in ref. [6]). For further details the reader be referred to ref. [6].

The $\pi \pi$-interaction in the pseudo-geminal phane 3 is expected to be stronger than in the pseudo-ortho phane 2 due to the difference in the geometrical arrangement. In the pseudo-geminal phane $\underline{3}$ equivalent $\mathrm{C}$-atoms of the monomers and hence $2 \mathrm{p}_{\mathrm{z}}$-orbitals with identical spin and charge density are situated opposite to each other in the two subunits. This leads to a higher total intersystem bond density $d_{i}^{\pi}$ than the configuration encountered in 2 where $2 \mathrm{p}_{\mathrm{z}}$-atomic orbitals with different charge densities are situated opposite to each other, for details the reader is referred again to [6].

In addition, a direct interaction between the oxygen atoms exists in the excited state of $\underline{3}$ which is absent in $\underline{2}$ and which leads to an additional bond density $d_{i j}^{\pi}$ where $i$ and $j$ refer to the oxygen atoms in the two subunits. The strength of this interaction depends on the degree of extension of the singly occupied molecular $\pi$-orbitals to the oxygen atoms. The situation is further complicated by the steric hindrance between the methoxy groups which is expected to be somewhat larger in the pseudo-geminal phane 3. We do not attempt at present to estimate the importance of the direct interaction between the methoxy groups.

The emission spectra of 2 and 3 are structureless broad bands. While this is in general found for the fluorescence of phanes which consist of two identical subunits [1-6], it is frequently not so in the case of the phosphorescence which is found to show a considerable amount of structure, for instance, in the cases of pyrenophane [4] and fluorenophane [5]. This difference in behaviour of the phosphorescence is due to the difference in distances between the two subunits in the various phanes. While the distance is about $3.6-3.8 \AA$ in the central part of the larger phanes mentioued above $[8,9]$, it is only about $3.1 \AA$ for the smaller paracyclophane [10] and for substituted paracyclophanes [11]. Although the excited triplet orbitals are less extended along the $z$-direction perpendicular to the plane of the aromatic subunits than the corresponding singlet orbitals $[1,6]$, their interaction, i.e. their intersystem bond density $d_{i}^{\pi}$, is still fairly large in the smaller phanes leading to red shifted broad structureless phosphorescence bands.

An analogous behaviour is also found for the zero field splitting parameters. The reduction of the $D$ parameter with respect to the monomer is $14 \%$ for 2 and $22 \%$ for 3 . This difference in reduction of $D$ is due to the stronger interaction in 
3 mentioned above which leads to a higher contribution of the charge transfer terms to the wave functions of the triplet electrons in $\underline{3}$ as compared to $\underline{2}$.

Striking differences are found in the decay rates $k_{i}$ of the phanes with respect to the monomer $\underline{l}$ and also between $\underline{2}$ and $\underline{3}$. The average decay rate $\rho$ for $\underline{2}$ is a factor of 10 , and for $\underline{3}$ a factor of 15 larger than for the monomer. Even more striking are the changes in the individual decay rates $k_{i}$. The decay rate $k_{x}$ for $\underline{3}$ is 50 times as large as the $k_{x}$ for the monomer 1 and still 7 times as large as $k_{x}$ for the pseudo-ortho phane 2 . These large ratios show that the decay constants which are very sensitive to changes in structure are suitable parameters to discriminate between stereoisomer [2.2]phanes as well as between [2.2]phanes and monomers.

It is tempting to compare the behaviour of the phanes with the predictions of a model which is frequently used for the excited triplet state of a pair of interacting molecules [12-17]. However, this is problematic because the $\pi$ electron interaction is rather strong in the phanes due to the shorter distance between the two subunits which are pressed together by the $\mathrm{CH}_{2}-\mathrm{CH}_{2}$ bridges. This stronger interaction leads to higher contributions of the charge transfer terms to the wave function of the excited triplet electrons and hence to a higher value of the total intersystem bond density $d_{1}^{\pi}$ as mentioned above [6]. Consequently, the simple model is not applicable since it is based on the assumption that the two triplet electrons are in the same subunit of the pair at a given time although this assumption is not always explicitly mentioned.

Our measurements show that the average decay rate $\rho$ is an experimentally accessible quantity in which this stronger interaction manifests itself. The stronger the $\pi$-electron interaction, the more important the charge transfer terms, the higher becomes $\rho$. This confirms a suggestion by Bowman and Norris [17] that the model of interacting pairs mentioned above is only applicable if the average decay rate $\rho$ is about the same for the pair as for the corresponding monomers.

\section{Acknowledgement}

We are very grateful to Professor J.P. Colpa for a detailed discussion of the manuscript.

\section{References}

[1] D. Schwettzer, J.P. Colpa, J. Behnke, K.H. Hausser, M.Haenel and H.A. Staab, Chem. Phys 11 (1975) 373.

[2] D. Schwettzer, J.P. Colpa, K.H. Hausser, M. Haenel and H.A. Staab, J. Lumınescence 12/13 (1976) 363.

[3] D. Schweitzer, K.H. Hausser, V. Taglieber and H.A. Staab, Chem. Phys. 14 (1976) 183.

[4] D. Schwettzer, K.H. Hausser, R.G.H. Kirrstetter and H.A. Staab, Z. Naturforsch. 31a (1976) 1189.

[5] D. Schweitzer, K.H. Hausser and M.W. Haenel, Chem. Phys. 29 (1978) 181. 
[6] J.P. Colpa, K.H. Hausser and D. Schweitzer, Chem. Phys. 29 (1978) 187.

[7] H.A. Staab and W. Rebafka, Chem. Ber. 110 (1977) 3333.

[8] H. Irngartinger, R.G.H. Kirrstetter, C. Krieger, H. Rodewald and H.A. Staab, Tetrahedron Lett. (1977) 425.

[9] H. Irngartinger, C. Krieger and H. Rodewald, to be published.

[10] D.K. Lonsdale, H.J. Milledge and K.V. Krishna Rao, Proc. Roy. Soc. London A 225 (1960) 82;

H. Hope, J. Bernstein and K.N. Trueblood, Acta Cryst. B 28 (1972) 1733.

[11] H. Irngartinger and B. Merkert, to be published.

[12] H. Sternlicht and H.M. McConnell, J. Chem. Fhys. 35 (1961) 1793.

[13] M. Schwoerer and H.C. Wolf, Mol. Cryst. 3 (1967) 177.

[14] R.M. Hochstrasser and T.S. Lin, J. Chem. Phys. 49 (1968) 4929.

[15] R.H. Clarke, R.E. Connors, H.A. Frank and J.C. Hoch, Chem. Phys. Lett. 45 (1977) 523.

[16] W. Hägele, D. Schmid and H.C. Wolf, Z. Naturforsch. 33a (1978) 94.

[17] M.K. Bowman and J.R. Norris, Chem. Phys. Lett. 54 (1978) 45. 